\title{
Face Recognition Based on Feature Fusion
}

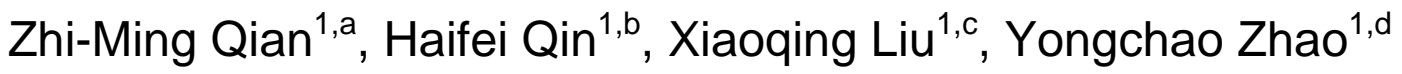 \\ ${ }^{1}$ Chuxiong Normal University, Chuxiong, China \\ aqzhiming@126.com, bqhfei@cxtc.edu.cn, cliuxiaoqing@cxtc.edu.cn, \\ dzhaoyongchao@cxtc.edu.cn
}

Keywords: Face recognition; Local feature; Gabor transform; Global feature.

\begin{abstract}
A face recognition method based on local feature and global feature is presented. Firstly, local feature of the face are extracted by Gabor wavelet transformation. Then, principal component analysis (PCA) is used to extract the global feature of the face. Finally, these features are integrated as the general features and the final result is given. The experiment shows that the performance of the proposed method is superior to the method based the single feature.
\end{abstract}

\section{Introduction}

As a biometrics identification technology based on face features, face recognition is able to recognize the identity of each person according to the face image by extracting the mode characteristics implied in the face image and comparing them with a given face. Compared with the traditional identity authentication method, face recognition is able to provide higher security, reliability and effectiveness, which makes it attract more and more attention from people and begin to be applied in every field of social life [1]. Although new face recognition technologies have arisen constantly in recent decades, face recognition is still one of the most difficult research topics in the field of biometric identification and even in the field of artificial intelligence due to the characteristics of the human face itself, it can be reflected specifically in the following two aspects: (a) Similarity: Since the difference between two individuals is very slight, then high degree of similarity can be observed between all of the face structures, even between the outlines of human faces. Under this circumstance, such a characteristic is always adverse to face recognition although it might be helpful to face detection. (b) Changeability: Face shape is changeable. Any change in human face will bring about lots of facial expressions. Visual face images might vary greatly when people are observed from different angles. Moreover, face recognition has been affected by multiple factors such as light condition, obstruction and age etc.

Currently, there are two main face recognition methods: one is based on the global feature and the other method is based on the local feature with the former mainly taking the overall face attributes into account and the latter mainly considering the attributes of the local block-based face image. Researches made in recent years prove that human eyes have experienced a hierarchical from-coarse-to-fine and from-overall-to-part processing procedure when people should perceive something through their eyes [2]. Then it would be very hard to solve the problem in face recognition just based on a certain feature. On account of this, now more and more researchers begin to focus on such a method integrated with global feature and local feature to improve the effect of face recognition $[3,4]$.

In this paper, a face recognition method fused with global and local features is proposed. It's able to extract separately the global feature and the local feature of the face through the principal component analysis (PCA) method and the Gabor transformation to obtain the recognition result by integrating the result of the global feature and that of the local feature. The experimental result on ORL face database proves that this method is superior to the other recognition methods, which are designed just based on a single global or local feature as far as the recognition effect is concerned. 


\section{Local feature}

Researches show that Gabor wavelet is very similar to the response made by the simple cells in human vision system to the visual stimulation. Characterized by excellent spatial locality and direction selectivity, Gabor wavelet is able to capture the spatial frequencies in multiple directions and the local structural feature in a local image region [5]. Also it has certain robustness in image brightness, contrast change and the changes in face pose. In addition to these, a 2D Gabor function is able to enhance the image edge and the underlying image feature such as the peak, the valley and the outline of ridge etc. That's to say, it will not only improve the following information that is considered as the critical area in the face, including the eyes, the nose and the mouth etc., but also enhance the local feature such as the black nevus, the fossette and the scars etc., making it become a possibility that when the face information is preserved, the local feature can be enhanced as well. All of these turn out that the 2D Gabor function makes for the recognition of different face images.

A 2D Gabor function can be defined as:

$$
\psi_{\mu, \nu}(z)=\frac{\left\|k_{\mu, \nu}\right\|}{\sigma^{2}} e^{\left(-\left\|k_{\mu, \nu}\right\|^{2}\|z\|^{2} / 2 \sigma^{2}\right)}\left[e^{i k_{\mu, \nu} z}-e^{-\sigma^{2} / 2}\right]
$$

where $v$ and $\mu$ define the scale and orientation of the Gabor wavelets, $z=(x, y)$ is the image coordinate of a given position and $k_{\mu v}$, which is the center frequency of the filter, determines the direction and scale of the Gabor function. Perform the sampling in 8 directions and 5 scales with the expression shown as below:

$$
\begin{aligned}
& k_{\mu, v}=k_{v} e^{i \phi_{\mu}} \quad k_{v}=k_{\max } / f^{v}, v \in\{0,1, \ldots, 4\}, k_{\max }=\pi / 2, f=\sqrt{2} \\
& \phi_{u}=\pi \mu / 8, \mu \in\{0,1 \ldots, 7\}, \sigma=2 \pi
\end{aligned}
$$

The Gabor feature of an image can be defined as the convolution of the input image and the Gabor function. Assume that $f(x, y)$ is the gray value of the input image, then define the convolution of image $f(x, y)$ and the Gabor function as:

$$
G(x, y, \mu, v)=f(x, y) * \psi_{\mu, v}(x, y)
$$

where $G(x, y, \mu, v)$ represents the convolution result of the $v$ - $\mu$ Gabor function ( $v$ is the scale and $\mu$ is the direction) on the point $(x, y)$.

The results of Gabor transformation consisting of the real part and the virtual part are able to reflect the phase information and the amplitude information. The Gabor phase information will vary periodically with the change of the spatial position, while the amplitude information that is able to reflect the energy spectrum of the image varies in a comparatively smooth and stable way. Therefore this paper adopts the transformed amplitude to describe the face features.

\section{Global feature}

As a feature extraction method that has been widely applied nowadays, the PCA method is able to extract the main face features in the face database through the $K-L$ expansion to construct an eigenface space so that the to-be-tested face image can be projected into the eigenface space in the recognition to obtain a group of projection coefficients for the matching with the various face images in the database [6]. Due to the effectiveness in the dimension reduction process and the face feature extraction, the PCA method has been widely applied in the field of face recognition. Actually face recognition process consists of two phases, including training and recognition through the PCA method.

First, vectorize the training sample matrix to indicate the training sample as:

$$
x=\left(x_{1}, x_{2}, \ldots, x_{M}\right)^{T}
$$

where vector $x_{i}$ is the $N^{*} M$-dimension column vector (with the size of each sample at $M^{*} N$ ) constituted by each column of vectors in the $i$-th image and having been deployed in a column, while $M$ is the number of the training samples. After that, calculate the average value of the training set, which is also the mean face vector: 


$$
\Psi=\frac{1}{M} \sum_{i=1}^{M} x_{i}
$$

Then calculate the difference between each face and the average face:

$d_{i}=x_{i}-\Psi, i=1,2, \ldots, M$

Finally, construct a covariance matrix:

$$
C=\frac{1}{M} \sum_{i=1}^{M} d_{i} d_{i}^{T}=\frac{1}{M} A A^{T}, \quad A=\left(d_{1}, d_{2}, \ldots, d_{M}\right)
$$

Calculate the eigenvalue of $\lambda_{i}$ and the eigenvector of $v_{i}$ according to the covariance matrix that has been constructed as above. Choose the maximum $p$ eigenvalues and the corresponding feature vectors according to the contribution rate of the eigenvalues to get:

$$
\varphi=\sum_{i=1}^{p} \lambda_{i} / \sum_{i=1}^{M} \lambda_{i} \geq \alpha
$$

Assume that $\alpha=95 \%$, it indicates that 95\% of energy will be produced when the training samples are projected into the first $p$ feature vector sets. Calculate the eigenvector of the original covariance matrix:

$$
u_{i}=A v_{i} / \sqrt{\lambda_{i}}, \quad(i=1,2, \ldots, p)
$$

Then the eigenface space can be expressed as:

$$
w=\left(u_{1}, u_{2}, \ldots, u_{p}\right)
$$

\section{Feature fusion}

For every to-be-identified face image, first conduct a Gabor transformation on the image to obtain 40 Gabor face images. Then segment each Gabor face image into $4 * 4$ blocks and integrate all of the sub-blocks with the same sequence number based on all of the block images to obtain a $40 * 4 * 4$ eigenmatrix. After that, reduce the dimensions of the eigenmatrix corresponding to each sub-block through the PCA method. Finally calculate the sum of all distances between the relevant eigenmatrixes that correspond to the various sub-blocks of the to-be-identified image and the eigenmatrixes corresponding to the different sub-blocks of the training image to get $L_{i}$, the local similarity. After the local feature is available, utilize the PCA method to extract the global feature. Meanwhile calculate the distance between the to-be-identified sample and the eigenmatrix of the training sample to get the global similarity, $G_{i}$. Finally integrate in parallel the global similarity and the local similarity according to the weighted summation method to obtain the final classification result:

$$
S=\omega L_{i}+(1-\omega) G_{i}
$$

where $\omega$ is the weight of local feature in face recognition with the algorithm flow shown in Figure 1.

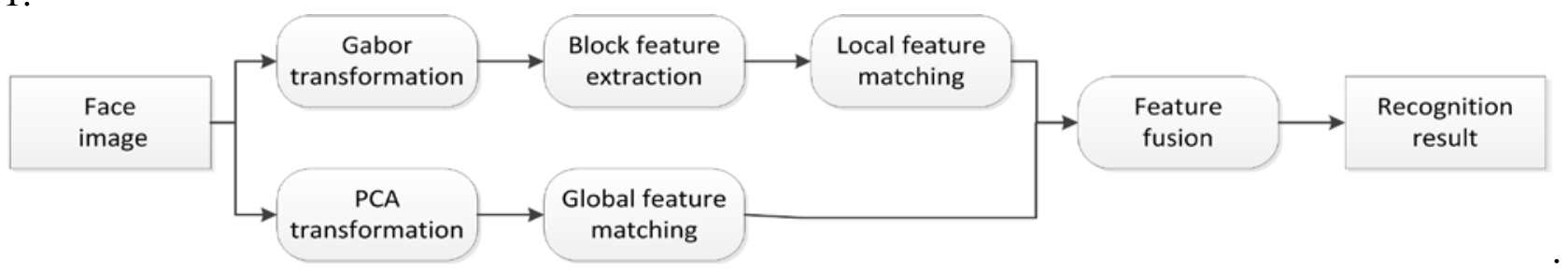

Figure 1. The flow chart of the proposed method.

\section{Experiments and analysis}

Data used in this experiment is from ORL face database involved with 40 persons and 10 images for each person [7]. With 256-level gray, each image contains $112 * 92$ pixels. All of the faces are in vertical and front view with certain changes in the illumination and face expression. For every person, 
conduct the experiment respectively by taking the first 4 face images in the face database as the training samples and the rest 6 images as the test samples.

In order to compare the performance of each method, this paper makes a contrast on our method, the PCA method based on global feature and the Gabor method based on local feature in the ORL face database. The experiment results are shown in Table 1, revealing that our face recognition method integrated with global and local features outperforms those methods that are established just based on single global feature or local feature in terms of the recognition result. All of these illustrate fully that regarding face recognition, the idea about the integration of global and local features to improve a face recognition system is effective, proving further the correctness of the estimation made by the biologists on human's recognition capacity.

Table 1. Performance comparisons among different methods on the ORL database.

\begin{tabular}{|l|l|l|}
\hline PCA & Gabor & The proposed method \\
\hline $87.5 \%$ & $90.8 \%$ & $93.3 \%$ \\
\hline
\end{tabular}

\section{Conclusion}

In this paper, a face recognition method integrated with global feature and local feature is proposed. It's able to extract the global feature of the face through the PCA method and at the same time extract the local feature of the face through the Gabor transformation to recognize the face images with the integration of both features. The experiment on ORL face database shows that although the global feature and the local feature play a different role in face recognition, the integration of both recognition results based on these two features does have contributed to the great improvement in the recognition rate of the system. All of these illustrate fully that the global feature and the local feature are highly complementary in improving the performance of the face recognition system.

\section{Acknowledgements}

This work was supported by the Scientific Research Fund of Yunnan Provincial Education Department (No. 2013 Y053 and No. 2012Y132), and the Program for Innovative Research Team (in Science and Technology) in University of Yunnan Province.

\section{References}

[1] Zhao, W., Chellappa, R., Phillips, P. J., \& Rosenfeld, A. (2003). Face recognition: A literature survey. Acm Computing Surveys (CSUR), 35(4), 399-458.

[2] Chellappa, R., Wilson, C. L., \& Sirohey, S. (1995). Human and machine recognition of faces: A survey. Proceedings of the IEEE, 83(5), 705-741.

[3] Su, Y., Shan, S., Chen, X., \& Gao, W. (2009). Hierarchical ensemble of global and local classifiers for face recognition. Image Processing, IEEE Transactions on, 18(8), 1885-1896.

[4] Liu, Z., \& Liu, C. (2010). Fusion of color, local spatial and global frequency information for face recognition. Pattern Recognition, 43(8), 2882-2890.

[5] Lee, T. S. (1996). Image representation using 2D Gabor wavelets. Pattern Analysis and Machine Intelligence, IEEE Transactions on, 18(10), 959-971.

[6] Turk, M., \& Pentland, A. (1991). Eigenfaces for recognition. Journal of cognitive neuroscience, 3(1), 71-86.

[7] Samaria F, Harter A. Parameterisation of a Stochastic Model for Human Face Identification. Proceeding of IEEE Workshop on Applications of Computer Vision. 1994, 138 142. 